\title{
Politics and conflicts
}

\begin{abstract}
:
By exploring some of the diverse theoretical literature on domestic politics and conflict, this research makes a first step toward bridging this divide. It looks at the link between national characteristics and war behavior, the possibility of democratic and non-democratic governments going to war, Marxist and liberal ideas on the role of economic structure, the impact of nationalism and public opinion, and the scapegoat theory. But first, this essay examines how political scientists and historians handle domestic origins of conflict.
\end{abstract}

Keywords: War, politics, behavior 


\section{Introduction}

Kwa kuchunguza baadhi ya fasihi anuwai ya nadharia juu ya siasa za nyumbani na mizozo, utafiti huu unachukua hatua ya kwanza kuelekea kuziba mgawanyiko huu. Inaangalia uhusiano kati ya sifa za kitaifa na tabia ya vita, uwezekano wa serikali za kidemokrasia na zisizo za kidemokrasia kwenda vitani, maoni ya Marxist na huria juu ya jukumu la muundo wa uchumi, athari ya utaifa na maoni ya umma, na nadharia ya mbuzi. Lakini kwanza, insha hii inachunguza jinsi wanasayansi wa kisiasa na wanahistoria wanavyoshughulikia asili ya ndani ya mizozo.

\section{Literature Review}

Kijadi, utafiti mwingi wa sayansi ya siasa juu ya vita umefuata dhana ya "mwanahalisi" na umezingatia muundo wa mfumo wa kimataifa na mwingiliano wa kimkakati kati ya majimbo kama vigezo vya msingi vya mzozo wa kimataifa. Katika miaka kumi na tano iliyopita kumekuwa na kuongezeka kwa hamu katika jukumu la vigeu vya urasimu-kisiasa na kisaikolojia katika michakato inayoongoza kwa vita, haswa katika fasihi juu ya uamuzi wa shida. Ingawa nadharia za kiuchumi za ubeberu na vita vimebuniwa na Wamarxist-Leninists, wanasayansi wa kisiasa kwa ujumla wamepunguza athari ya moja kwa moja ya anuwai ya kiuchumi kwenye michakato inayoongoza kwa vita. Hivi karibuni kumekuwa na kuongezeka kwa umakini kwa jukumu la mambo ya uchumi, ingawa lengo limekuwa haswa juu ya athari ya mabadiliko ya uchumi kwa viwango tofauti vya ukuaji wa kitaifa na mabadiliko yanayosababishwa katika usambazaji wa nguvu za kijeshi kimataifa.

Ingawa masomo ya mapema hayakupata uhusiano thabiti kati ya aina ya utawala na tabia ya vita, hivi karibuni kumekuwa na nia mpya katika pendekezo la Kantian kwamba demokrasia asili yake ni ya amani na kwamba serikali zisizo za kidemokrasia ni za vita zaidi. Hoja ya kimsingi ya Kant ni kwamba katika serikali ya jamhuri (inayojulikana na katiba, serikali ya uwakilishi na mgawanyo wa madaraka) raia wanatawala, na "wale ambao watalazimika kuamua kunyimwa vita vyote watasita kuanza uovu kama huo mchezo. " Waamuzi katika nchi ambazo sio za kidemokrasia wana uwezekano mkubwa wa kushiriki katika vita, hata "kwa sababu ndogo" kwa sababu wao wenyewe hawapati moja kwa moja matokeo yake ya kibinadamu na kwa sababu hawazuiliwi na mfumo wa ukaguzi na mizani au uwajibikaji wa uchaguzi.

Ingawa maelezo kadhaa ya kuaminika ya kukosekana kwa vita kati ya demokrasia yamependekezwa, hakuna iliyojaribiwa kwa ukali na kimfumo. Hitimisho moja la busara, hata hivyo, ni kwamba maelezo ya muundo tu, ambayo 
hayatofautishi kati ya majimbo kwa msingi wa tabia zao za ndani, hayawezi kuhesabu tofauti za kitabia zilizozingatiwa kati ya nchi za kidemokrasia na zisizo za kidemokrasia. Jibu labda liko katika vigeuzi vya ndani kwa majimbo.

Kuna maoni mengi ya mshikamano wa nadharia na uhalali wa kihistoria wa nadharia ya Marxist-Leninist ya ubeberu; vidokezo vichache vitatosha hapa. Kwanza, hata ikiwa mtu angekubali uhusiano kati ya ubepari na ubeberu, uhusiano wa kinadharia kati ya ubeberu na vita, haswa vita vya kati, haujawahi kuonyeshwa kwa kusadikisha. Inawezekana pia kuwa upanuzi wa kibeberu, haswa katika enzi ya mpaka wazi wa kikoloni, hupunguza uwezekano wa vita kuu kwa kugeuza ushindani mkubwa wa nguvu kutoka msingi wa mfumo kwenda pembezoni, ambapo masilahi yao muhimu hayana uwezekano wa kugongana na ambapo suluhisho za maelewano zinawezekana zaidi. Kautsky alipendekeza kuwa ushindani wa kibeberu utasababisha "ubeberu mkubwa," ushirikiano kati ya majimbo ya kibepari kwa unyonyaji wa pamoja wa pembezoni. Pili, kwa kiwango cha kijeshi, ikiwa tunachukulia uhusiano madhubuti kati ya mifumo ya kisiasa ya kidemokrasia ya huria na mifumo ya uchumi wa kibepari, nadharia ya Marxist-Leninist inafanya utabiri mbili ambao unapingana moja kwa moja na uhusiano wa kijeshi kati ya demokrasia huria na vita. Vita vilivyotabiriwa kati ya majimbo ya kibepari huria hayakuwa ya kawaida, na majimbo ya kibepari hayajawahi kukabiliwa na vita au uwezekano mkubwa wa kuanzisha vita kuliko majimbo mengine katika mfumo wa kimataifa..

Watu katika nchi zote za kidemokrasia na zisizo za kidemokrasia mara nyingi huwa na shauku mwanzoni mwa vita, ingawa msaada huu unaweza kupungua haraka ikiwa vita vitaendelea na kugharimu. Katika siasa za Amerika uungwaji mkono maarufu kwa rais unakua kila mara baada ya matumizi ya nguvu, bila kujali hekima au mafanikio ya hatua hiyo ya kijeshi. Mfano huu umeelezewa na tabia ya umma ya kuzunguka bendera, rais, na chama, na mwishowe na hali ya utaifa wa kisasa.

Kinadharia, nadharia ya Azazeli inategemea nadharia ya kikundi / nje ya kikundi katika sosholojia. Simmel, katika matibabu ya kimfumo ya kwanza ya mada hiyo, alisema kuwa mzozo na kikundi cha nje huongeza mshikamano na ujumuishaji wa kisiasa wa kikundi hicho, na jumla kwa uhusiano wa kimataifa: "vita na nje wakati mwingine ndio nafasi ya mwisho kwa serikali iliyojaa uhasama wa ndani kushinda haya maelewano, au vinginevyo kuvunja dhahiri.

" Coser alibadilisha maoni mengi ya Simmel. Anasema kuwa mshikamano wa kikundi hicho utaongezwa tu ikiwa tayari kuna kiwango kidogo cha mshikamano wa ndani na ikiwa tu inagunduliwa kuwa tishio la nje linahatarisha kikundi kwa ujumla na sio sehemu yake tu. Vinginevyo, mzozo wa nje utasababisha mzozo wa ndani na utengano 
badala ya mshikamano. Coser ni mamlaka iliyotajwa sana juu ya nadharia ya kikundi / nje ya kikundi, lakini sifa hii muhimu haitambuliki kila wakati.

Maelezo ya kimsingi ya kukosekana kwa ushahidi wa uhusiano kama huo ni kukosekana kwa mfumo wa nadharia uliokua vizuri unaoongoza masomo ya kijeshi. Sababu moja ya umaskini huu wa kinadharia ni mipaka nyembamba sana ya nidhamu na kutofaulu kwa wanasayansi wa kisiasa kuthamini vyanzo vyenye utajiri wa ufahamu wa nadharia katika nyanja zingine. Ingawa wanasayansi wa kisiasa mara nyingi wanakubali utumiaji unaofaa wa fasihi za kihistoria za kupima ujasusi wao wa nadharia, wanadharau matumizi yake kama chanzo cha mapendekezo ya nadharia. Fasihi zinazohusiana na nadharia ya mbuzi ni mfano bora wa hoja ya Bueno de Mesquita kwamba "mara nyingi hatulete ukali sana kwa nadharia yetu kama tunavyofanya kwa uchambuzi wetu wa data," lakini pia inaonyesha vyanzo vingi vya ufahamu muhimu wa nadharia ambayo inaweza kusaidia katika kazi ya maendeleo ya kinadharia.

\section{Conclusion}

Kwa kuchunguza baadhi ya fasihi anuwai ya nadharia juu ya siasa za nyumbani na mizozo, utafiti huu unachukua hatua ya kwanza kuelekea kuziba mgawanyiko huu. Inaangalia uhusiano kati ya sifa za kitaifa na tabia ya vita, uwezekano wa serikali za kidemokrasia na zisizo za kidemokrasia kwenda vitani, maoni ya Marxist na huria juu ya jukumu la muundo wa uchumi, athari ya utaifa na maoni ya umma, na nadharia ya mbuzi. Lakini kwanza, insha hii inachunguza jinsi wanasayansi wa kisiasa na wanahistoria wanavyoshughulikia asili ya ndani ya mizozo. 


\section{References}

Hang, N. K., L., Huong, H. T. T., Huong, N. T., Kien, L., \& Khoi, N. D. (2021). The Long-run Effects of War: A Literature Review. https://doi.org/10.31219/osf.io/kg39r

Le, K. (2020). Land use restrictions, misallocation in agriculture, and aggregate productivity in Vietnam. Journal of Development Economics, 145, 102465.

Huong, H. T. T., Hang, N. K., L., Khoi, N. D., Kien, L., \& Huong, N. T. (2021). A Literature Review on the Impacts of Armed Conflicts on Human Development. https://doi.org/10.31219/osf.io/mg4d6

Le, K. (2021a). Extending Maternity Leave and Early Childhood Health in Zimbabwe. Review of International Geographical Education Online, 11(5), 4276-4282.

Khoi, N. D., L., Huong, H. T. T., Huong, N. T., Hang, N. K., \& Kien, L. (2021). The Effects of Climate Extremes on Health: A Literature Review. https://doi.org/10.31219/osf.io/es5zt

Le, K. (2021b). Armed Conflict and Child Weight in DR Congo. Advances in Public Health, 2021.

Nguyen, M. (2018). The Relationship between Race-Congruent Students and Teachers: Does Racial Discrimination Exist?. Available at SSRN 3305463.

Charney, E., Kitzman, H., Berkow, E., Cafarelli, C., Davis, L., Disney, F., ... \& Miller, R. (1971). The childhealth nurse (pediatric nurse practitioner) in private practice: a controlled trial. New England Journal of Medicine, 285(24), 1353-1358.

Le, K., \& Nguyen, M. (2019). 'Bad Apple' peer effects in elementary classrooms: the case of corporal punishment in the home. Education Economics, 27(6), 557-572.

Trang, T. L., Huong, N. T., Khoi, N. D., Huong, H. T. T., Hang, N. K., \& Kien, L. (2021). The Consequences of Nutrition Hazards: A Literature Review. https://doi.org/10.31219/osf.io/wutbv

Le, K., \& Nguyen, M. (2020a). Aerial bombardment and educational attainment. International Review of Applied Economics, 34(3), 361-383.

Haggerty, R. J., Roghmann, K. J., \& Pless, I. B. (Eds.). (1975). Child health and the community. Transaction Publishers.

Le, K., \& Nguyen, M. (2020b). The impacts of farmland expropriation on Vietnam's rural households. Review of Development Economics, 24(4), 1560-1582.

Gutelius, M. F., Kirsch, A. D., MacDonald, S., Brooks, M. R., \& McErlean, T. (1977). Controlled study of child health supervision: Behavioral results. Pediatrics, 60(3), 294-304.

Le, K., \& Nguyen, M. (2020c). Armed conflict and birth weight. Economics \& Human Biology, 39, 100921.

Nguyen, M. (2021). Mask Mandates and COVID-19 Related Symptoms in the US. ClinicoEconomics and Outcomes Research: CEOR, 13, 757.

Morley, D., \& Woodland, M. (1979). See how they grow-monitoring child growth for appropriate health care in developing countries. Macmillan Press Ltd., 4 Little Essex Street, London WC2R 3LF..

Le, K., \& Nguyen, M. (2020d). Shedding light on maternal education and child health in developing countries. World Development, 133, 105005.

Larson, C. P. (1980). Efficacy of prenatal and postpartum home visits on child health and development. Pediatrics, 66(2), 191-197.

Le, K., \& Nguyen, M. (2021a). In-utero Exposure to Rainfall Variability and Early Childhood Health. World Development, 144, 105485.

Acheson, R. M. (1954). A method of assessing skeletal maturity from radiographs: a report from the Oxford Child Health Survey. Journal of Anatomy, 88(Pt 4), 498. 
Stouffer, S. A., Suchman, E. A., DeVinney, L. C., Star, S. A., \& Williams Jr, R. M. (1949). The american soldier: Adjustment during army life.(studies in social psychology in world war ii), vol. 1.

Le, K., \& Nguyen, M (2021b). The Impacts of Temperature Shocks on Birth Weight in Vietnam. Population and Development Review.

Merton, R. K., Fiske, M., \& Curtis, A. (1946). Mass persuasion; the social psychology of a war bond drive.

McLuhan, M., \& Fiore, Q. (1968). War and peace in the global village.

Le, K., \& Nguyen, M. (2021c). The psychological consequences of COVID-19 lockdowns. International Review of Applied Economics, 35(2), 147-163.

Singer, J. D., Bremer, S., \& Stuckey, J. (1972). Capability distribution, uncertainty, and major power war, 1820-1965. Peace, war, and numbers, 19(48), 427.

Le, K., \& Nguyen, M. (2021d). The psychological burden of the COVID-19 pandemic severity. Economics \& Human Biology, 41, 100979.

World Health Organization. (1978). A growth chart for international use in maternal and child health care: guidelines for primary health care personnel. World Health Organization.

Le, K., \& Nguyen, M. (2021e). Education and political engagement. International Journal of Educational Development, $85,102441$.

Hewitt, D., Westropp, C. K., \& Acheson, R. M. (1955). Oxford Child Health Survey: effect of childish ailments on skeletal development. British journal of preventive \& social medicine, 9(4), 179.

Lifton, R. J. (1973). Home from the war: Vietnam veterans: Neither victims nor executioners.

Le, K., \& Nguyen, M. (2021f). How Education Empowers Women in Developing Countries. The BE Journal of Economic Analysis \& Policy, 21(2), 511-536.

Stuart, H. C., \& Reed, R. B. (1959). Longitudinal studies of child health and development. Series 2. Description of project. Pediatrics, 24(5), 875-885. 